\title{
Gamma photometric redshifts for long gamma-ray bursts
}

\author{
Z. Bagoly ${ }^{1}$, I. Csabaii ${ }^{2}$, A. Mészáros ${ }^{3}$, P. Mészáros ${ }^{4}$, I. Horváth ${ }^{5}$, L. G. Balázs ${ }^{6}$, and R. Vavrek ${ }^{7}$ \\ ${ }^{1}$ Laboratory for Information Technology, Eötvös University, 1117 Budapest, Pázmány P. s. 1./A, Hungary \\ e-mail: bagoly@ludens.elte.hu \\ 2 Dept. of Physics for Complex Systems, Eötvös University, 1117 Budapest, Pázmány P. s. 1./A, Hungary \\ e-mail: csabai@ludens.elte.hu \\ 3 Astronomical Institute of the Charles University, V Holešovičkách 2, 18000 Prague 8, Czech Republic \\ e-mail: meszaros@mbox.cesnet.cz \\ ${ }^{4}$ Dept. of Astronomy \& Astrophysics, Pennsylvania State University, 525 Davey Lab., University Park, PA 16802, USA \\ e-mail: nnp@astro.psu.edu \\ 5 Dept. of Physics, Bolyai Military University, 1456 Budapest, POB 12, Hungary \\ e-mail: hoi@jkmf.hu \\ ${ }^{6}$ Konkoly Observatory, 1505 Budapest, POB 67, Hungary \\ e-mail: balazs@ogyalla.konkoly.hu \\ 7 Max-Planck-Institut für Astronomie, 69117 Heidelberg, 17 Königstuhl, Germany \\ e-mail: vavrek@mpia-hd.mpg.de
}

Received 28 June 2002 / Accepted 18 November 2002

\begin{abstract}
It is known that the soft tail of the gamma-ray bursts' spectra show excesses from the exact power-law dependence. In this article we show that this departure can be detected in the peak flux ratios of different BATSE DISCSC energy channels. This effect allows to estimate the redshift of the bright long gamma-ray bursts in the BATSE Catalog. A verification of these redshifts is obtained for the $8 \mathrm{GRB}$ which have both BATSE DISCSC data and measured optical spectroscopic redshifts. There is good correlation between the measured and estimated redshifts, and the average error is $\Delta z \approx 0.33$. The method is similar to the photometric redshift estimation of galaxies in the optical range, hence it can be called as "gamma photometric redshift estimation". The estimated redshifts for the long bright gamma-ray bursts are up to $z \simeq 4$. For the the faint long bursts - which should be up to $z \simeq 20$ - the redshifts cannot be determined unambiguously with this method.
\end{abstract}

Key words. cosmology: large-scale structure of Universe - gamma-rays: bursts

\section{Introduction}

The gamma-ray bursts (hereafter GRBs) of the long subgroup (Kouveliotou et al. 1993) detected by the BATSE instrument (Meegan et al. 2000) are at high redshifts. The highest directly measured redshift is at $z=4.5$ (Andersen et al. 2000; Mészáros 2001), but there are indirect considerations - based on BATSE data - predicting the existence of redshifts up to $z \simeq 20$ (Mészáros \& Mészáros 1995, 1996; Horváth et al. 1996; Balázs et al. 1998). This result is based on distribution densities and deals with the GRB redshifts in statistical sense only. This means that one may obtain the fraction of GRBs being at a given redshift interval (see, for example, Schmidt 2001), but one cannot obtain the redshift of a given GRB event.

There are only a few cases, when the observation with the BeppoSAX satellite (Piro et al. 2002) or other instruments (Klose 2000) made possible to detect the afterglows and then the measurement of redshifts using optical spectroscopy.

Send offprint requests to: Z. Bagoly, e-mail: bagoly@ludens.elte.hu
The Current BATSE Catalog (Meegan et al. 2000) consists of more than 1200 long bursts, but for only 9 of them have redshift measurement ( 8 have redshifts and there is one GRB with an upper redshift limit). The unfortunate premature termination of the CGRO satellite prevents to increase this number further. There are other instruments observing $\approx 100$ bursts/year, but the typical number of burst's redshifts is only about a dozen/year (Mészáros 2001).

Hence, any method that could estimate the redshifts from X-ray/gamma-ray observations alone would be a great help.

In Ramirez-Ruiz \& Fenimore (2000) and Reichart et al. (2001) a linear relation between the intrinsic peak-luminosities of GRBs and their so called "variabilities" was found. Similarly, Norris et al. (2000a) found a relation between the so called spectral lag and the peak-luminosity allowing to estimate the redshifts of long GRBs. These relations were calibrated on a few cases of GRBs, when GRBs were observed both by BATSE and other instruments measuring the optical redshift from afterglows. Then, having either the variabilities or the spectral lag of a given GRB, one can estimate its redshift. 
The physical meaning of the correlation between the variability (spectral lag) and the peak-luminosity remains unclear. These two methods can be combined (Schaefer et al. 2001) to determine redshifts if all the needed input parameters are available for the GRBs.

In this article we present a new method of the estimation of the redshifts for the long GRBs. The situation is in some sense similar to the optical observations of galaxies, where the number of objects with broad band photometric observations is much larger than the number of objects with measured spectroscopic redshifts. For galaxies and quasars the growing field of photometric redshift estimation (Koo 1985; Connolly et al. 1995; Gwyn \& Hartwick 1996; Sawicki et al. 1997; WangBahcall et al. 1998; Fernández-Soto et al. 1999; Benítez 2000; Csabai et al. 2000; Budavári et al. 2000; Budavári et al. 2001) achieved a great success in estimating redshifts from photometry only. Here we present a method that is quite similar to these methods; hence we call it as gamma photometric redshift estimation (GPZ for short). We utilize the fact that broadband fluxes change systematically, as characteristic spectral features redshift into, or out of the observational bands. Hence, contrary to the variability and spectral lag methods, this technique has a well defined physical meaning.

The article is structured as follows. First, using a spectral model for GRBs we deduce an expected relation between a measurable quantity (peak flux ratio) and the redshift (Sect. 2). Having this relation, we verify it on the existing sample of a few GRBs having measured redshifts (Sect. 3). Because both Sects. 2 and 3 suggest that this method is usable, Sect. 4 presents the estimated redshifts for hundreds of long GRBs. In Sect. 5 we discuss and summarize the results.

\section{Gamma photometric redshift estimation}

To understand our method in this section we outline the general scheme of broadband observations. The method is generally the same both for the optical and gamma-ray ranges. The only major difference is that in the X-ray and gamma-ray range the extra- and intergalactic medium have negligible effects, but the optical photons are attenuated.

Let us take two different instrumental channels defined by $E_{4}>E_{3}$ and $E_{2}>E_{1}$. If one would know the rest-frame energy spectrum $(L(E))$ of the burst, for a perfect instrument that captures all the photons in the above energy channels, the observed luminosity (in units photons/s) for a burst at redshift $z$ would be the following:

$L_{2,1}=\int_{(1+z) E_{1}}^{(1+z) E_{2}} L(E) \mathrm{d} E, \quad L_{4,3}=\int_{(1+z) E_{3}}^{(1+z) E_{4}} L(E) \mathrm{d} E$.

For the observed fluxes $(P)$ similar equations can be used. Let us define the following flux ratio:

$R\left(E_{4}, E_{3}, E_{2}, E_{1}, z\right)=\frac{L_{4,3}-L_{2,1}}{L_{4,3}+L_{2,1}}=\frac{P_{4,3}-P_{2,1}}{P_{4,3}+P_{2,1}}$

which in general depends on the redshift, since $L_{2,1}$ and $L_{4,3}$ depends on the redshift.
Assume for the moment that one observes a pure powerlaw spectrum. This means that $L_{E} \propto E^{-\alpha}$ holds, where the exponent $\alpha$ is a real number. In this special case one could prove easily that for any redshift $z$

$\frac{L_{4,3}^{z=0}-L_{2,1}^{z=0}}{L_{4,3}^{z=0}+L_{2,1}^{z=0}}=\frac{L_{4,3}-L_{2,1}}{L_{4,3}+L_{2,1}}$,

where

$L_{2,1}^{z=0}=\int_{E_{1}}^{E_{2}} L(E) \mathrm{d} E, \quad L_{4,3}^{z=0}=\int_{E_{3}}^{E_{4}} L(E) \mathrm{d} E$.

This means that in this special case $R=R\left(E_{4}, E_{3}, E_{2}, E_{1}, z\right)$ is not depending on $z$.

Of course, in the real situation, the spectrum has got a more complicated form, and hence $R$ is depending on $z$; this will be the effect that we will use for redshift estimation.

In addition, in the real situation, the incident spectrum measured by the detector is convolved with the detector's response function defined by response matrix (Pendleton et al. 1994) resulting the measured flux of the corresponding channel. For the channel with energy range $E_{2}>E>E_{1}$ the measured flux $P_{1,2}$ is therefore given by

$P_{1,2}=\int_{E_{1}}^{E_{2}} P(E) c(E) \mathrm{d} E$,

where $c(E)$ is the detector's response function. The similar holds for the second channel, too, with the same $c(E)$. Hence, in general, $R$ is depending both on the spectrum and the response function.

If the rest-frame spectrum for a GRB is known, one is able to calculate the theoretical $R$ as a function on $z$. Then these values can be compared with the flux ratio obtained from the broadband measurements $\left(R_{\text {meas }}\right)$. The redshift, where $\left(R-R_{\text {meas }}\right)^{2}$ is minimal, could give the estimated gamma photometric redshift.

Regarding this gamma photometric redshift estimation, the major problem comes from the fact that the spectra are changing quite rapidly with time; the typical timescale for the time variation is $\simeq(0.5-2.5) \mathrm{s}$ (Ryde \& Svensson 1999, 2000). Hence, if possible, one should consider spectra which are defined for time intervals smaller than this characteristic time. Therefore, we will consider the spectra in the $320 \mathrm{~ms}$ time interval (i.e. in five $64 \mathrm{~ms}$ time intervals), with the peak-flux being at the center of this time interval.

In the following we will assume that the spectrum has the same shape around the time of the peak-flux for all long bursts. Unfortunately we do not have any deep theoretical or observational evidence for this assumption, instead we will test our assumption on GRBs, where spectroscopic redshifts are available (next section). Because, this assumption seems to be acceptable, in Sect. 4 we will use $R$ to estimate $z$ for long GRBs.

\section{Application on GRBs: Calibration}

It is well-known (Band et al. 1994; Amati et al. 2002) that the time-integrated average spectra of GRBs can be approximated by a broken power-law; the break is at some energy $E_{\mathrm{o}}$. 
The typical rest-frame energy for $E_{\mathrm{o}}$ is above $\simeq 500 \mathrm{keV}$ (Preece et al. 2000a; Preece et al. 2000b), but this might vary for different GRBs.

Of course this broken power-law spectrum is simply an approximation: first, because the break around $E_{0}$ may have a more complicated form (Preece et al. 2000a; Preece et al. 2000b), and, second, because at low rest-frame energies (around $\simeq 80 \mathrm{keV}$ ) there may be essential departures from the power-law. This is the so called soft-excess, which is confirmed for $\simeq 15 \%$ of GRBs on the high confidence level (Preece et al. 1996, 2000a; Preece et al. 2000b); and for the remaining GRBs the soft-excess seems to occur, too (Preece et al. 1996).

Based on this, we construct our template spectrum that will be used in the GPZ process in the following manner: Let the spectrum be a sum of the Band's function (Band et al. 1994), and of a low energy power-law function taking the form

for $E \leq E_{\mathrm{o}} \quad L(E)=a\left(E / E_{\mathrm{cr}}\right)^{-\alpha}+a\left(E / E_{\mathrm{cr}}\right)^{-\beta}$

for $E \geq E_{\mathrm{o}} L(E)=a_{3}\left(E / E_{\mathrm{o}}\right)^{-\gamma}$,

where $a_{3}=a\left[\left(E_{\mathrm{o}} / E_{\mathrm{cr}}\right)^{-\alpha}+\left(E_{\mathrm{o}} / E_{\mathrm{cr}}\right)^{-\beta}\right]$ comes from the normalization. In this spectrum there are six parameters, but the amplitude $a$ is for $R$ unimportant, and need not be specified. We fix all the above parameters according to the available literature, so there are no free tunable parameters in our method. The low energy cross-over is at $E_{\mathrm{cr}}=90 \mathrm{keV}, E_{\mathrm{o}}=500 \mathrm{keV}$, and the spectral indices are $\alpha=3.2, \beta=0.5$ and $\gamma=3.0$ (Preece et al. 2000a; Preece et al. 2000b).

As we remarked above, the spectrum is rapidly changing. But our assumption is that the spectrum has a characteristic shape around the instant of the peak. Now we have to chose a short time interval around the peak (maximum of the total counts), during which the change of spectrum is still negligible, but the number of photons allows good signal-to-noise ratio. To be able to cut out such time interval around the peak-flux, we need data with a reasonably good time resolution. In our study we will use the $64 \mathrm{~ms}$ resolution BATSE LAD DISCSC data from the public BATSE Catalog (Meegan et al. 2000). During this time interval the change of our template spectrum is still negligible (Ryde \& Svensson 2000).

We have also checked the robustness of the PFR against the integration time around the peak. Both the doubling of the integration time (for $640 \mathrm{~ms}$ ) and its skewing around the peak did not change significantly the values of PFR. All this means that the template spectrum defined by Eqs. (6), (7) seems to be a good approximation for the $320 \mathrm{~ms}$ time interval around the peak. This is in fact expectable from earlier studies of spectra (Band et al. 1994; Ryde \& Svensson 1999, 2000).

The 4 energies for the BATSE instrument are: $E_{1}=25 \mathrm{keV}$, $E_{2}=E_{3}=55 \mathrm{keV}, E_{4}=100 \mathrm{keV}$. Using the detector response matrices (Pendleton et al. 1994) one can calculate the observed counts and flux for any incoming spectrum. In Fig. 1 we show a typical response function $c(E)$. The response function is different for each burst, but using the BATSE DRM data one can use the actual response function for every burst. Figure 1 also demonstrates the behaviour of the spectrum at different redshifts. Going from $z=0$ to higher redshifts one can see that the soft-excess moves from the second channel to the first one and then leaves the range of this detector around $z \approx 4$.

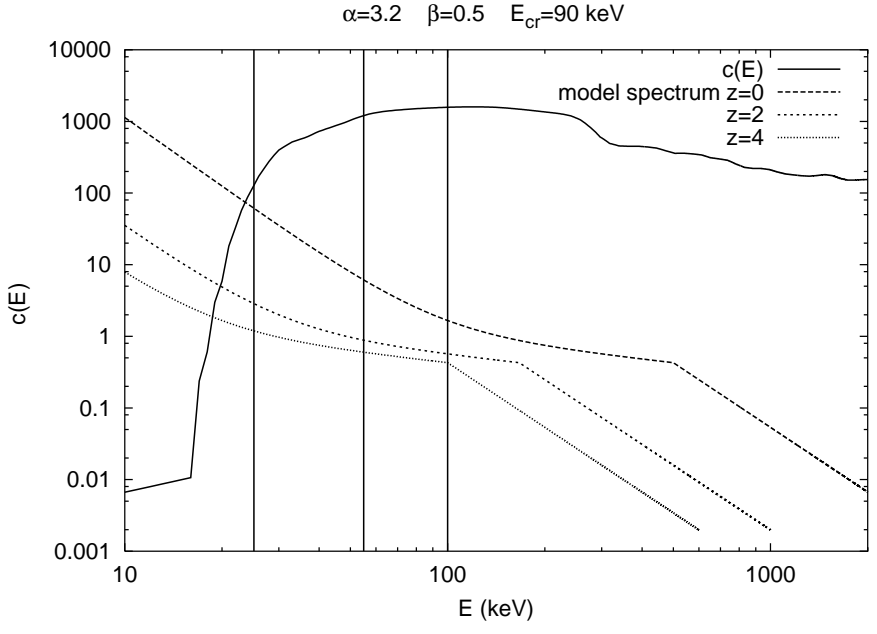

Fig. 1. The detector response function $c(E)$ and the behaviour of a template spectrum at different redshifts.

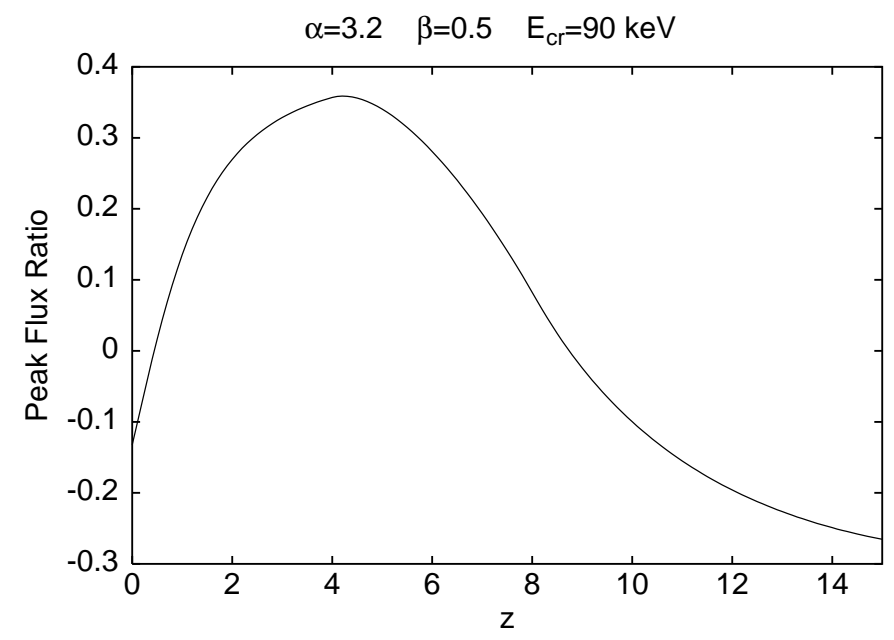

Fig. 2. The theoretical PFR curves calculated from the template spectrum using the average detector response matrix.

Before starting the detailed investigation of the fluxes that one can get using the template spectra and the response matrices by Eq. (6), let us test the correctness of the template spectrum in a simple way. Let us introduce the peak flux ratio (PFR hereafter) in the following way:

$\mathrm{PFR}=\frac{l_{34}-l_{12}}{l_{34}+l_{12}}$

where $l_{i j}$ is the BATSE DISCSC flux in energy channel $E_{i}<$ $E<E_{j}$ integrated for $320 \mathrm{~ms}$ around the peak flux. (I.e. five $64 \mathrm{~ms}$ intervals are summed - the middle one is where the flux is the biggest. Even during this time interval the change of spectrum is still negligible (Ryde \& Svensson 2000).) Our theory says that for the above template spectrum this ratio should increase with $z$. In Fig. 2 we plot the theoretical PFR curves calculated from the above defined template spectrum using the average detector response matrices for the 9 bursts that have both BATSE data and measured redshifts. These bursts' data are collected in Table 1.

If we redshift the template spectrum and apply the corresponding detector response matrix of the given burst, we can 
Table 1. The redshifts of GRBs that have both BATSE triggers and measured spectroscopic redshifts. Data compiled by Klose (2000); see also Bloom et al. (2001).

\begin{tabular}{llll}
\hline \hline Burst & $\begin{array}{l}\text { BATSE } \\
\text { trigger }\end{array}$ & Remark \\
\hline 980425 & 6707 & 0.00857 & SN1998bw \\
970508 & 6225 & 0.8356 & \\
970828 & 6350 & 0.9578 & no DISCSC data \\
980703 & 6891 & 0.9676 & \\
991216 & 7906 & 1.020 & \\
990123 & 7343 & 1.6006 & \\
990510 & 7560 & 1.6196 & \\
971214 & 6533 & 3.4127 & \\
980329 & 6665 & 3.5 & upper limit only \\
\hline
\end{tabular}

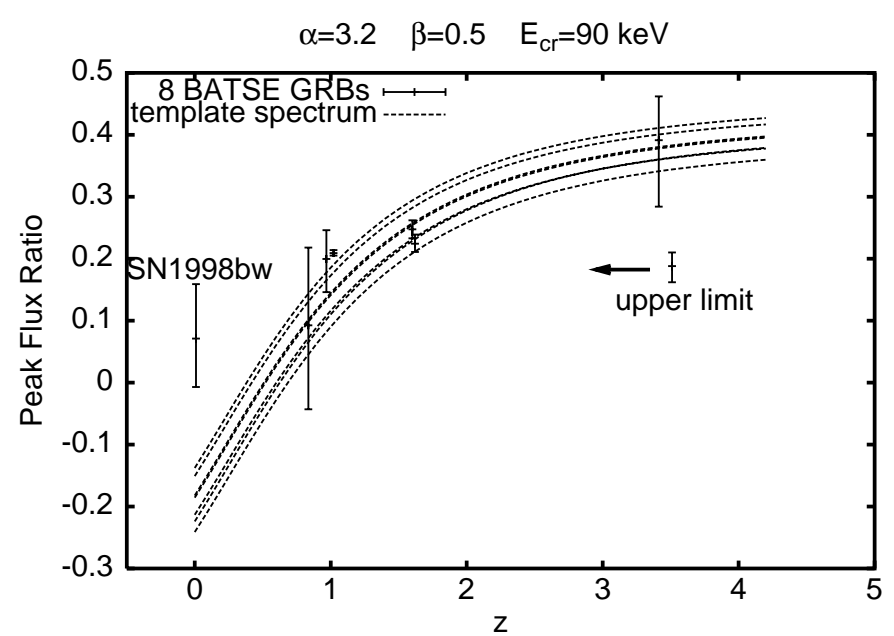

Fig. 3. Experimental (points with error bars) and theoretical (dashed lines) peak flux ratio values for the 8 bursts that have both BATSE DISCSC data and measured redshifts.

get a PFR value for any redshift. Figure 3 shows the theoretical curves together with the seven PFR values calculated from observed GRB data (the DISCSC data for GRB 970828 are missing). We used the template spectrum defined by Eqs. (6), (7) and the different response matrices corresponding to the observational conditions of the given burst. There is a clear trend: as expected from the above considerations, PFR increases with increasing redshifts up to $z \simeq 4$. Except for the GRB associated with the supernova, and for the GRB having only the upper limit, the remaining 6 GRBs have a clearly increasing PFR with increasing $z$.

In the used range of $z$ (i.e. for $z \lesssim 4$ ) the relation between $z$ and PFR is invertable. Hence we can use it to estimate the gamma photometric redshift (GPZ) from a measured PFR. In Fig. 4 the measured spectroscopic redshifts are compared with GPZ values for 8 considered GRBs. The errorbars show the effect of counts' Poisson noise only.

Leaving out GRB associated with the supernova and GRB having upper redshift limit only, the estimation error is $\Delta z=\sqrt{\sum_{i=1}^{6}\left(z_{i}^{\mathrm{spec}}-z_{i}^{\mathrm{GPZ}}\right)^{2} / 5} \approx 0.33$.

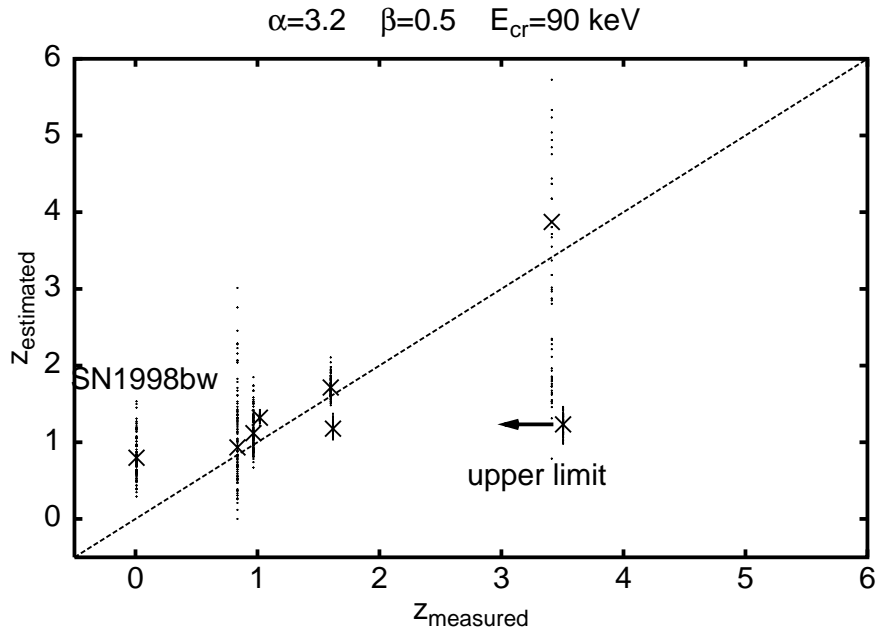

Fig. 4. The measured spectroscopic redshift values compared with gamma photometric redshift estimation for the 8 considered GRBs.

Table 2. The linear correlation coefficients between $z^{\text {spec }}$ and $z^{\mathrm{GPZ}}$.

\begin{tabular}{cccc}
\hline \hline$N$ & $r_{\mathrm{c}}$ & $p=1-\beta$ & Remark \\
\hline 7 & 0.9364 & 0.9991 & \\
6 & 0.9655 & 0.9991 & GRB 980425 excluded \\
6 & 0.7809 & 0.9666 & GRB 971214 excluded \\
5 & 0.6618 & 0.8881 & GRB 980425 + GRB 971214 excluded
\end{tabular}

In order to test the reality of the correlation between the soft excess and the redshift we made the null hypothesis that there is no relationship between these quantities, i.e. the computed correlation is purely random. Assuming no true correlation between the soft excess and redshift the probability density of the computed quantity can be given by

$f(x)=\frac{1}{\sqrt{\pi}} \frac{\Gamma((N-1) / 2)}{\Gamma((N-2) / 2)}\left(1-x^{2}\right)^{(N-4) / 2}$,

where $N$ is number of data points (Spiegel \& Stephens 1999). Let $r_{\mathrm{c}}$ be the calculated correlation. Then the $\beta$ error probability at rejecting the null hypothesis is given by

$\beta=1-\int_{-1}^{r_{c}} f(x) \mathrm{d} x$.

In Table 2 the $N, r_{\mathrm{c}}$ and the calculated level of significance are shown for various cases.

Although it seems that $z^{\mathrm{GPZ}}$ for GRB 971214 (where $z=$ 3.4127) fits very well the estimation error without it is better: $\Delta z \approx 0.29$. However the linear correlation coefficient here with $N=5$ yields a much poorer $r_{\mathrm{c}}=0.66$ with a $p=0.89$ significance.

We see that PFR (if calculable from observations for the given burst) is a quantity that may allow to determine redshift. Problems may arise from the fact that for any value of PFR two redshifts are possible - either below or above $z \simeq 4$ (see Fig. 2), further measurements are needed to exclude one of the redshift. 


\section{Application on GRBs: Estimation of the redshifts}

To avoid the problems with the instrumental threshold we exclude the faintest GRBs from the BATSE data. Similarly to Pendleton et al. (1997) and Balázs et al. (1998) these events have a $F_{256}$ peak-flux (i.e. on $256 \mathrm{~ms}$ trigger scale) smaller than 0.65 photon $/\left(\mathrm{cm}^{2} \mathrm{~s}\right)$. These GRBs are not discussed in this article.

Further restriction comes from the fact that short GRBs are today taken as different phenomena (Horváth et al. 2000; Norris et al. 2000b). In addition, due to instrumental effects (Piro et al. 2002), no spectroscopic redshifts are known for this subgroup of GRBs. Hence, we do not apply our method for short GRBs.

The reality of the intermediate subgroup of GRBs (Horváth 1998; Mukherjee et al. 1998; Hakkila et al. 2000; Balastegui et al. 2001; Rajaniemi et al. 2002; Horváth 2002) having remarkable sky angular distribution (Mészáros et al. 2000a,b; Litvin et al. 2001) is unclear yet. In any case, no spectroscopic redshifts are known also here. Hence, we exclude this subgroup, too.

Therefore, we restrict ourselves to long GRBs defined by $T_{90}$ (Meegan et al. 2000) with $T_{90}>10 \mathrm{~s}$. There are 1241 GRBs in BATSE Catalog fulfilling this condition. Deleting GRBs having no $F_{256}$ and having $F_{256}<$ 0.65 photon $/\left(\mathrm{cm}^{2} \mathrm{~s}\right) 838$ GRBs remain. This sample is studied here.

Introducing an another cut $F_{256}>2.00$ photon/ $\left(\mathrm{cm}^{2} \mathrm{~s}\right)$ we can investigate roughly the brighter half of this sample. We will discuss the sample $F_{256}>2.00$ photon/( $\mathrm{cm}^{2} \mathrm{~s}$ ) ("bright half" sample having $343 \mathrm{GRBs})$ and $F_{256}>0.65$ photon $/\left(\mathrm{cm}^{2} \mathrm{~s}\right)$ ("all" sample having $838 \mathrm{GRBs}$ ), respectively.

As the soft-excess range redshifts out from the BATSE DISCSC energy channels around $z \approx 4$, the theoretical curves converge to a constant value. For higher $z$ it starts to decrease. This is where the power-law breakpoint $\left(E_{\mathrm{o}}\right)$ is redshifts into soft energy range. This means that the method is ambiguous: for the given value of PFR one may have two redshifts - below and above $z \approx 4$. Because for the bright GRBs the values above $z \approx 4$ are practically excluded, for them the method is usable. In other words, using only the $25-55 \mathrm{keV}$ and $55-100 \mathrm{keV}$ BATSE energy channels, this method can be used to estimate GPZ only in the redshift range $z \lesssim 4$; outside of this region the $z$ vs. PFR relation is non-invertable (see Fig. 2). For high redshifts (above $z \approx 4$ ) the method gives two possible values. For faint GRBs the estimation also usable (at least in principle), but one has to decide by other arguments that either the redshift below $z \approx 4$ or above $z \approx 4$ is the correct value.

Let us assume for a moment that all observed long bursts, we have selected above, have $z<4$. Then we can simply calculate the $z^{\mathrm{GPZ}}$ redshift for any GRB, which has calculable PFR from BATSE DISCSC data. Figure 5 shows the distribution of the measured PFRs of the long GRBs having DISCSC data. The fact that the number of objects beyond the minimal and maximal theoretical PFR values $(\approx-0.15$ and $\approx 0.37$, respectively) is relatively small, is reassuring.

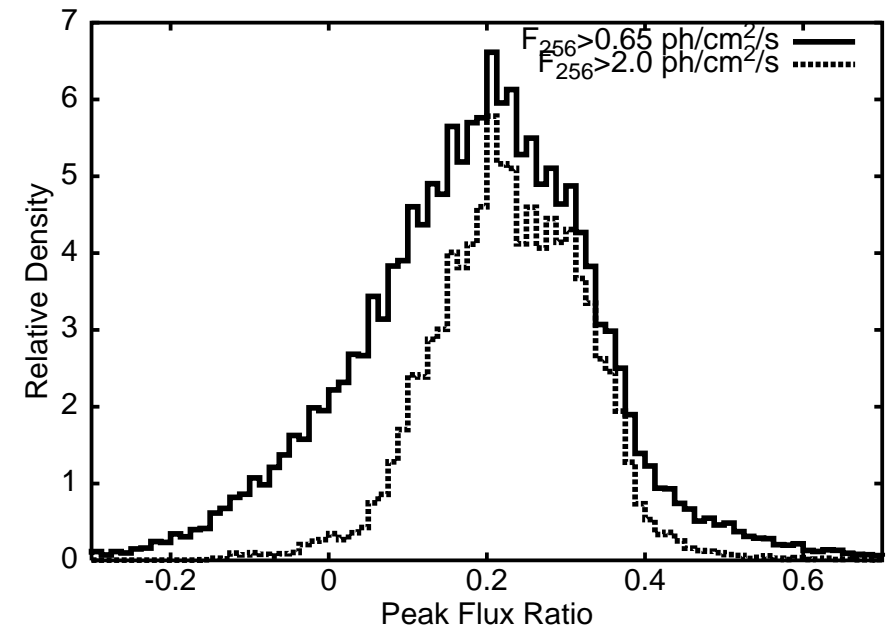

Fig. 5. The PFR distribution of the long GRBs having DISCSC data.

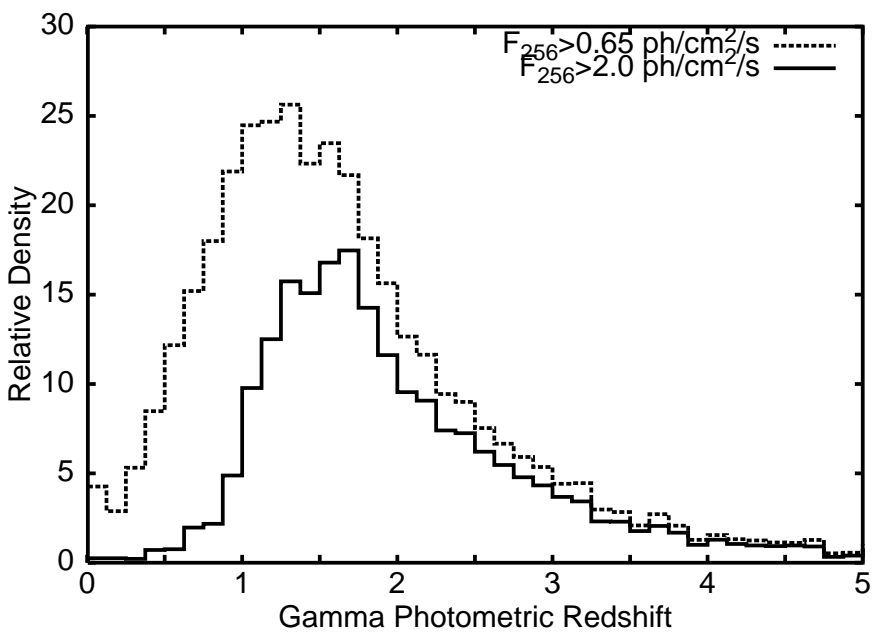

Fig. 6. The distribution of the Gamma Photometric Redshift estimators of the long GRBs having DISCSC data. The distribution of the bright half of the BATSE Catalog is also shown.

Figure 6 shows the distribution of the estimated derived redshifts under the assumption that all GRBs are below $z \approx 4$. The distribution has a clear peak value around $\mathrm{PFR} \approx 0.2$, which corresponds to $z \approx(1.5-2.0)$.

\section{Discussion}

Having the estimated redshifts shown in Fig. 6 one may ask: Are these redshifts really correct?

There can be two different problems here. First of all, the method is based on the assumption that around the peak flux, the spectrum is the same for all the selected long GRBs. Second, the method gives degenerate result, with two possible redshift values.

Concerning the first problem we could just hope that in the near future some theoretical or experimental evidence will confirm our assumption, but the situation is not worse than in the studies of Norris et al. (2000a) and Reichart et al. (2001). These articles also suggest that despite the deeper understanding of the underlying physics, the procedure itself is usable. In addition, here the PFR- $z$ relation is well supported by earlier independent observations. 


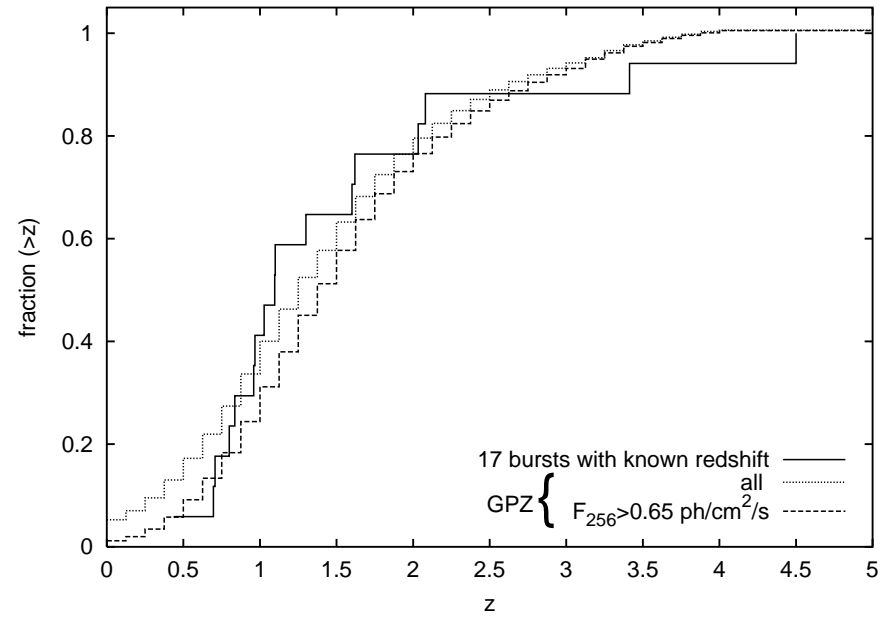

Fig. 7. The redshift distribution of the 17 GRBs' with known redshift and the distributions from the Gamma Photometric Redshift estimators for different peak flux groups.

Concerning the second problem we think that the great majority of values of $z$ obtained for the bright half are correct. This opinion may be supported by three independent arguments. First, the obtained distribution of GRBs in $z$ for the bright half in Fig. 6 is very similar to the obtained distribution of Schmidt (2001) (see Fig. 6 of that article). The luminosity-based redshift distribution (Schaefer et al. 2001) also suggest an uniformly rising GRB density out to $z \approx 5$. Second, as $z$ moves into $z \gtrsim 4$ regime for the bright GRB, one would obtain extremely high luminosities. Using Eq. (12) of Mészáros \& Mészáros (1996), there is a lower limit for the isotropic luminosity of the GRBs a value $\simeq 10^{53} \mathrm{ergs} / \mathrm{s}$. (Note here that the precise value is, of course, calculable and is depending on the chosen cosmology model and on the typical energy of emitted photons. For the purpose of this article this approximate value is enough.) This is an unacceptable high value for a lower limit, because typical luminosities are $\simeq 10^{51-52} \mathrm{ergs} / \mathrm{s}$ (see, e.g., Table 1 of Reichart et al. 2001). We cannot exclude that a few cases from bright GRB are at $z \gtrsim 4$, but - we think - in the bright half this cases are rare. Thirds, as an additional statistical test we compared the redshift distribution of the 17 GRB with observed redshift with our reconstructed GRB $z$ distributions (limited to the $z<4$ range). For the $F_{256}>0.65$ photon $/\left(\mathrm{cm}^{2} \mathrm{~s}\right)$ group the Kolmogorov-Smirnov test suggests a $38 \%$ probability, i.e. the observed $N(<z)$ probability distribution agrees quite well with the GPZ reconstructed function. Although the observed distribution suffers from strong selection effects this fact is nevertheless reassuring.

For the faint GRBs being between $F_{256}=$ 0.65 photon $/\left(\mathrm{cm}^{2} \mathrm{~s}\right)$ and $F_{256}=2.00$ photon $/\left(\mathrm{cm}^{2} \mathrm{~s}\right)$ the situation is different. From Fig. 6 it follows that for $z<1.7$ GRBs should be dominated by faint objects. From this figure one would obtain that GRBs are in average at smaller redshifts. This is clearly a wrong conclusion, which is caused by the false assumption that $z<4$ for all the faint GRBs. We think that the majority of faint GRBs $z$ should be changed into the value $z \gtrsim 4$. Unfortunately, we are not able to say, concretely which GRB has a great $(z \gtrsim 4)$, and which GRB has still a small $(z \lesssim 4)$ redshift. In addition, for these faint GRBs also the error of estimated redshifts should probably be bigger than $\Delta z \approx 0.33$. Simply, we conclude that in the current form with the current data, our method is not applicable for the faint GRBs.

The results of this work may be summarized as follows.

1. Based on earlier observations of GRB spectra it is shown that the peakflux ratio (PFR) should be a well defined function of $z$.

2. The estimated redshifts from PFR are in good accordance with the known redshifts of the few GRBs in BATSE Catalog having spectroscopic redshifts

3. All this allows us to calculate the redshifts of long GRBs. Unfortunately, due to the twofold character of the PFR curve, the method is usable only for bright GRBs.

4. Redshift distribution of 343 bright long GRBs are determined (Fig. 6).

Acknowledgements. The useful remarks with Drs. T. Budavári, S. Klose, D. Reichart, A. S. Szalay and the anonymous referees are kindly acknowledged. This research was supported in part through OTKA grants T024027 (L.G.B.), F029461 (I.H.) and T034549, Czech Research Grant J13/98: 113200004 (A.M.), NASA grant NAG5-9192 (P.M.).

\section{References}

Amati, L., Frontera, F., Tavani, M., et al. 2002 [astro-ph/0205230] Andersen, M. I., Hjorth, J., Pedersen, H., et al. 2000, in Gamma-Ray Bursts in the Afterglow Era, Proc. Int. Workshop, Held in Rome, Italy, 17-20 October 2000, ed. E. Costa, F. Frontera, \& J. Hjorth (Springer), ESO Astrophysics Symp., 133

Balázs, L. G., Mészáros, A., \& Horváth, I. 1998, A\&A, 339, 1

Balastegui, A., Ruiz-Lapuente, P., \& Canal, R. 2001, MNRAS, 328, 283

Band, D. L., Ford, L. A., Matteson, J. L., et al. 1994, ApJ, 434, 560

Benítez, N. 2000, ApJ, 536, 571

Bloom, J. S., Kulkarni, S. R., \& Djorgovski, S. G. 2001, AJ, 123, 1111

Budavári, T., Szalay, A. S., Connolly, et al. 2000, AJ, 120, 1588

Budavári, T., Csabai, I., Szalay, A. S., et al. 2001, AJ, 122, 1163

Connolly, A. J., Csabai, I., Szalay, A. S., et al. 1995, AJ, 110, 2655

Csabai, I., Connolly, A. J., Szalay, A. S., et al. 2000, AJ, 119, 69

Fernández-Soto, A., Lanzetta, K. M., \& Yahil, A. 1999, ApJ, 513, 34

Gwyn, S. D. J., \& Hartwick, F. D. A. 1996, ApJ, 468, L77

Hakkila, J., Haglin, D. J., Pendleton, G. N., et al. 2000, ApJ, 538, 165

Horváth, I., Mészáros, P., \& Mészáros, A. 1996, ApJ, 470, 56

Horváth, I. 1998, ApJ, 508, 757

Horvath, I. 2002, A\&A, 392, 791

Horváth, I., Balázs, L. G., \& Mészáros, P., et al. 2000, in Gamma-Ray Bursts in the Afterglow Era, Proc. Int. Workshop, Held in Rome, Italy, 17-20 October 2000, ed. E. Costa, F. Frontera, \& J. Hjorth (Springer) ESO Astrophysics Symp., 66

Koo, D. C. 1985 , AJ, 90, 148

Kouveliotou, C., Meegan, C. A., \& Fishman, G. J. 1993, ApJ, 413, L101

Klose, S. 2000, Rev. Mod. Astron. 13, Astronomische Gesellschaft, Hamburg, 129

Litvin, V. F., Matveev, S. A., Mamedov, S. V., et al. 2001, Pis'ma v Astronomicheskiy Zhurnal, 27, 495

Meegan, C., Malozzi, R. S., Six, F., \& Connaughton, V. 2000, Current BATSE Gamma-Ray Burst Catalog, http://gammaray.msfc.nasa.gov/batse/grb/catalog 
Mészáros, A., \& Mészáros, P. 1996, ApJ, 466, 29

Mészáros, A., Bagoly, Z., \& Vavrek, R. 2000a, A\&A, 354, 1

Mészáros, A., Bagoly, Z., Horváth, I., et al. 2000b, ApJ, 539, 98

Mészáros, P., \& Mészáros, A. 1995, ApJ, 449, 9

Mészáros, P. 2001, Science, 291, 79

Mukherjee, S., Feigelson, E. D., Babu, G. J., et al. 1998, ApJ, 508, 314

Norris, J. P., Marani, G. F., Bonnell, J. T., et al. 2000a, ApJ, 534, 248

Norris, J. P., Scargle, J. D., \& Bonnell, J. T. 2000b, in Gamma-Ray Bursts in the Afterglow Era, Proc. Int. Workshop, Held in Rome, Italy, 17-20 October 2000, ed. E. Costa, F. Frontera, \& J. Hjorth (Springer) ESO Astrophysics Symp., 40

Pendleton, G. N., Paciesas, W. S., Briggs, M. S., et al. 1994, ApJ, 431, 416

Pendleton, C. N., Paciesas, W. S., Briggs, M. S., et al. 1997, ApJ, 489, 175

Piro, L., Gandolfi, G., Grandi, P., et al. 2002, http://www .ias.rm.cnr.it/ias-home/sax

Preece, R. D., Briggs, M. S., Pendleton, G. N., et al. 1996, ApJ, 473, 310
Preece, R. D., Espley, J. R., \& Briggs, M. S. 2000, in Gamma-Ray Bursts, 5th Huntsville Symp., 18-22 October 1999, ed. R. M. Kippen, R. S. Mallozzi, \& G. J. Fishman (Melville - New York), AIP Conf. Proc., 526, 175

Preece, R. D., Briggs, M. S., Mallozzi, et al. 2000, ApJS, 126, 19

Rajaniemi, H. J., \& Mahonen, P. 2002, ApJ, 566, 202

Ramirez-Ruiz, E., \& Fenimore, E. E. 2000, ApJ, 539, 712

Reichart, D. E., Lamb, D. Q., Fenimore, E. E., et al. 2001, ApJ, 552, 57

Ryde, F., \& Svensson, R. 1999, ApJ, 512, 693

Ryde, F., \& Svensson, R. 2000, ApJ, 529, L13

Sawicki, M. J., Lin, H., \& Yee, H. K. C. 1997, AJ, 113, 1

Schaefer, B. E., Deng, M., \& Band, D. L. 2001, ApJ, 563, L123

Schmidt, M. 2001, ApJ, 552, 36

Spiegel, M. R., \& Stephens, L. J. 1999, Schaum's Outline of Theory and Problems of Statistics, 3rd ed. (McGraw-Hill, New York)

Wang, Y., Bahcall, N., \& Turner, E. L. 1998, AJ, 116, 2081 